female gender $(\mathrm{OR}=1.61, P<0.001)$ and life satisfaction level (OR for range $=7.73, P<0.001)$.

Conclusions: The prevalence of self-reported headache among high school children in Zagreb city is relatively high; significant sex difference was observed. Pain among children and adolescents is an important public health problem.

43

\section{Management of patients with headache presenting to a neurological emergency room}

V. Vuković ${ }^{1}$, M. Knežević-Pavlić ${ }^{2}$, J. Tumpić-Jaković ${ }^{3}$, M. Strineka', A. Lovrenčić-Huzjan ${ }^{1} \&$ V. Demarin ${ }^{1}$

'University Hospital "Sestre Milosrdnice", Department of Neurology, Zagreb, Croatia, ${ }^{2}$ General Hospital Našice, Department of Neurology, Našice, Croatia, ${ }^{3}$ General Hospital Virovitica, Department of Neurology, Virovitica, Croatia

Introduction: Patients with headache frequently seek help in a neurological emergency room. In this study we tried to analyse the management of patients presenting with a headache to the emergency room (ER) at the University Hospital "Sestre milosrdnice" in Zagreb.

Methods: We have retrospectively analysed all patients with headache who were examined in our emergency room during 2007. Patient data were analyzed according to the diagnoses, diagnostic procedures, treatment and further refferal.

Results: Among 6225 patients, $1385(22.3 \%)$ complained of headache; $894(64.9 \%)$ women and $491(35.4 \%)$ men. Migraine with or without aura, tension-type headache or cervicogenic headache had $1009(72.9 \%)$ of patients $(67.3 \%$ women and $32.7 \%$ men); $84(6 \%)$ had intracranial haemorrhage, $33(2.3 \%)$ had primary tumour, $54(3.8 \%)$ metastases, $193(13.9 \%)$ head trauma, $7(0.5 \%)$ head trauma with heamorrhage and $5(0.4 \%)$ had an infective disease. A diagnostic procedure was indicated in 413 (29.8\%) of patients: $314(22.7 \%)$ had a CT scan, 85 (6.1\%) an EEG and $70(5 \%)$ had an ultrasound examination. Patient refferal was as following: $1022(73.8 \%)$ was dismissed home, $222(16 \%)$ was reffered to other clinics and $141(10.2 \%)$ was hospitalized. Among patients with primary and cervicogenic headaches a diagnostic procedure was performed in $235(23.2 \%)$ while $45(4.5 \%)$ was hospitalized.

Conclusions: Patients with primary headaches frequently seek help in the ER. For patients with primary headaches, better treatment should be provided by GPs and neurologists (headache specialists) in out-patient headache clinics. Mass media campaignes should be carried out in order to bring closer the possibilities of treatment for primary headaches.

\section{4}

Assessment of breath holding index during orthostasis P. Bago Rožanković1', A. Lovrenčić-Huzjan², M. Strineka², M. Crnjaković ${ }^{2} \&$ V. Demarin ${ }^{2}$

'University Hospital Dubrava, Department of Neurology, Zagreb,

${ }^{2}$ University Hospital Sestre Milosrdnice, Clinical Department of Neurology, Zagreb, Croatia

Introduction: The study was to assess the differences of cerebrovascular reactivity in healthy subjects during orthostasis. Subjects and methods: Twenty healthy volunteers $(11$ men and 9 women) with no atherosclerotic risk factors were evaluated by means of transcranial Doppler. A breath holding index (BHI) was obtained in supine and standing position using standardized procedure. Student's $t$-test was used for intergroup comparison of mean blood flow velocities (MBFV) and BHI values between supine and standing position and for left to right assessment.

Results: Mean blood flow velocity (MBFV) in a middle cerebral artery in supine position was $66.6 \mathrm{~cm} / \mathrm{s}$ on the right side and $68.5 \mathrm{~cm} / \mathrm{s}$ on the left side, and in standing position $60.6 \mathrm{~cm} / \mathrm{s}$ on the right side, $62.3 \mathrm{~cm} / \mathrm{s}$ on the left side. There was no significant difference in values of MBFV comparing supine and standing position and also between males and females. Mean BHI in supine position was 1.59 on the right side, 1.65 on the left side, and in standing position 1.63 on the right side, 1.7 on the left side, without significant difference comparing males and females. There was no statistically significant differences in the BHI between supine and standing position $(P=0.81$ for the right side, $P=0.68$ for the left side) neither between sides in supine $(P=0.71)$ and standing position $(P=0.8)$.

Conclusion: There was no significant difference in BHI values during orthostatic stress in evaluation of cerebrovascular reactivity.

45

\section{Median nerve dimensions measured using high- resolution ultrasound in healthy volunteers}

D. Azman, R. Bene, M. Strineka, J. Bosnjak, A. Huzjan-Lovrencic, M. Budisic \& V. Demarin

University Department of Neurology, Sestre Milosrdnice University Hospital, Reference Center for Neurovascular Disorders and Reference Center for Headache of the Ministry of Health and Social Welfare of the Republic of Croatia, Vinogradska 29, Zagreb, Croatia

Objectives: Although electroneuro- and electromyography are still leading diagnostic methods for investigation of peripheral nerves function, they do not provide information regarding their morphology. This study was conducted to evaluate the suitability of ultrasonography in visualization of median nerve in healthy volunteers.

Methods: Twenty-five asymptomatic volunteers (16 women and 9 men) have participated in this study, age ranging from 20 68 years.

Device used was Aloka Prosound Alpha10 Premier with $13 \mathrm{MHz}$ probe, using custom preset for musculo-skeletal sonography. Following dimensions of median nerve at the level of pisiform bone were measured bilaterally: cross-sectional area (CSA), circumference, longer and shorter radius. Subsequently, using latter values, flattening ratio was calculated.

Examinees' height was measured and handedness ascertained. Additional epidemiological data taken was the average daily time that individuals spent working on a personal computer as a possible factor for compression of the nerve in examinee's dominant hand.

Results: Median nerve was easily depicted in all of the participants as well as the surrounding soft-tissue structures. Average CSA of median nerve was $9.67 \mathrm{~mm}^{2}$ (range $5-15 \mathrm{~mm}^{2}$, with standard deviation of $2.4 \mathrm{~mm}$ ). Mean flattening-ratio (FR) (longer radius/ shorter radius) was 4.18 , ranging from 2.16 to 5.92 .

Median height was $173.8 \mathrm{~cm}$ and only one subject was left-handed while the others $(96 \%)$ were right hand dominant. Average daily time spent working on a personal computer (total average of 2.96 hours) did not correlate with CSA or FR values for the dominant hand.

Additionally, in two subjects, an aberrant artery accompanying $n$. medianus was visualized.

Conclusion: High-resolution sonographic imaging allows assessment of various morphological properties of median nerve, including its various dimensions and echoic architecture. Furthermore, ultrasound imaging is a very convenient (available, quick, inexpensive and noninvasive) method for examination of peripheral nerve morphology and could thus be used to enhance diagnostic efficiency. 